\title{
Parametric level statistics in random matrix theory: Exact solution
}

\author{
E. Kanzieper \\ The Abdus Salam International Centre for Theoretical Physics, P.O.B. 586, 34100 Trieste, Italy
}

(November 18, 1998)

\begin{abstract}
An exact solution to the problem of parametric level statistics in non-Gaussian ensembles of $N \times N$ Hermitian random matrices with either soft or strong level confinement is formulated within the framework of the orthogonal polynomial technique. Being applied to random matrices with strong level confinement, the solution obtained leads to emergence of a new connection relation that makes a link between the parametric level statistics and the scalar two-point kernel in the thermodynamic limit.
\end{abstract}

PACS number(s): 05.40.Ac, 02.10.Sp, 05.40.-a

In recent years, the theory of non-Gaussian random matrix ensembles has experienced a sound progress motivated by new ideas 近 in quantum chromodynamics (QCD) and mesoscopic physics. Both fields, concentrating on physically different objects which besides have incommensurable spatial scales, make use of the same language of random matrix theory [2] to reveal the universal statistical manifestations of symmetries inherent in a physical system. In QCD, invariant non-Gaussian random matrix models incorporating global symmetries of the QCD Dirac operator appear to describe the universal features [3] of low-energy part of the spectrum of the Dirac equation. In mesoscopic physics, similar matrix models of appropriate symmetries arise in identifying the universal electron level statistics in normalsuperconducting hybrid structures [4], as well as in the context of mesoscopic electron transport [5]. A new class of non-Gaussian random matrices [6] with finite level compressibility should also be mentioned; these serve as a basis for constructing the toy models [7] of universal spectral statistics expected at the edge of the metal-insulator transition.

One of the most promising ways of studying the nonGaussian random matrix ensembles consists of revealing the universal connection relations (i) between different entities (statistics) within the same matrix model, and (ii) between the same statistics in different matrix models (which only differ by underlying symmetries). In both cases the connection identities (if they exist) originate from underlying mathematical structure of random matrices which deserves to be studied in its own right, also. Being brought together, these two approaches have led to a substantial breakthrough in understanding the universality phenomenon in invariant random matrix ensembles characterized by strong level confinement [8].

(i) The Shohat method of Refs. [9, 10] is a typical example of the first approach. This formalism, employing the free fermion representation of the joint distribution function of eigenvalues in unitary invariant random matrix ensembles, reduces the problem of finding the $n$-point spectral correlators in the thermodynamic limit to a simpler problem of solving an effective oneparticle Schrödinger equation. In the absence of a fine tuning of confinement potential to so-called multicritical points [11], this equation involves two ingredients: the macroscopic mean density of states $\nu_{N}(E)$ (which is a one-point spectral characteristics) and the term determined by the singularities of confinement potential. In this way, one relates the $n$-point spectral characteristics to the one-point characteristics.

(ii) Recent studies by Şener and Verbaarschot [12], and by Widom 13 are representatives of the second approach. These authors, by using the two completely different constructions (of skew 14 and ordinary 15 orthogonal polynomials, respectively) succeeded in deriving universal relations between eigenvalue correlations in a class of non-Gaussian random matrix ensembles with the same, finite polynomial, confinement potential but with different symmetries. These remarkable relations express the spectral correlation functions of matrix models with orthogonal $(\beta=1)$ and symplectic $(\beta=4)$ symmetries in terms of the scalar two-point kernel of the corresponding ensemble with unitary symmetry $(\beta=2)$.

Contrary to conventional spectral statistics, which has received a detailed study in the literature, quite a bit is known 16,17] about parametric level statistics in nonGaussian random matrix models. In the present Letter, we address this problem for ensembles with unitary symmetry, simultaneously with taking a further step in establishing the connection relations in non-Gaussian random matrix theory. Previously, the issue of parametric correlations in non-Gaussian random matrix ensembles (on the scale of several mean level spacings) has been considered only by Hackenbroich and Weidenmüller [17] who used a supersymmetry formalism to provide a heuristic proof that in all three symmetry classes $(\beta=1,2$ and 4) the parametric spectral correlations of all orders in invariant matrix model given by Eq. (1) below are insensitive to the particular form of the confinement potential $v\left[\mathbf{H}_{0}\right]$, and thus follow the predictions found for Gaussian ensembles. This statement is valid for strong level confinement and refers to the bulk of the spectrum. Until now, however, no constructive formalism was proposed to quantitatively describe parametric level statistics in a random matrix ensemble with a general non-Gaussian measure. Our aim here is to formulate an exact solution 
to the problem of parametric level statistics for $\beta=2$. Our study differs from Refs. 16.17] in three main respects: (i) It applies to both strong and soft level confinement; (ii) it is valid in arbitrary spectrum range; (iii) it gives a prescription of how to actually compute parametric statistics for both arbitrary matrix size $N$ and in the thermodynamic limit. As an illustration, we apply the formalism developed to obtain closed analytical expressions for parametric eigenlevel correlations in the model Eq. (11) for strong level confinement in the origin, the bulk, and the soft-edge scaling limits [10].

Now we turn to the formalization of the problem and to the statement of the main results. Consider an unperturbed matrix ensemble defined by the joint distribution function of the entries of $N \times N$ Hermitian matrix $\mathbf{H}_{0}$,

$$
\mathcal{P}_{1}\left[\mathbf{H}_{0}\right]=\mathcal{Z}_{N}^{-1} \exp \left\{-\operatorname{Tr} v\left[\mathbf{H}_{0}\right]\right\} .
$$

Here $v\left[\mathbf{H}_{0}\right]$ is an arbitrary (not necessarily strong) confinement potential ensuring existence of the partition function $\mathcal{Z}_{N}$. Eigenlevel correlations of arbitrary order in this ensemble are known [2] to be expressed through the scalar two-point kernel $K_{N}\left(E, E^{\prime}\right)=$ $\sum_{n=0}^{N-1} \psi_{n}(E) \psi_{n}\left(E^{\prime}\right)$. Here the fictitious wave functions $\psi_{n}(E)=\mathcal{N}_{n}^{-1 / 2} \exp \{-v(E) / 2\} p_{n}(E)$ involve a set of polynomials $p_{n}(E)$ orthogonal on the entire real axis $\mathbf{R}$ with respect to the measure $d \mu(E)=\exp \{-v(E)\} d E$,

$$
\int d \mu(E) p_{n}(E) p_{m}(E)=\mathcal{N}_{n} \delta_{n m}
$$

In the presence of the perturbation, which we parameterize by the strength $\phi$, the Hamiltonian of the system modeled by the matrix $\mathbf{H}_{0}$ flows to $\mathbf{H}_{\phi}=\mathbf{H}_{0}+$ $\phi \mathbf{H}_{1}$. We choose the perturbation matrix $\mathbf{H}_{1}$ to belong to the Gaussian Unitary Ensemble (GUE), $\delta \mathcal{P}\left[\mathbf{H}_{1}\right] \propto$ $\exp \left\{-\operatorname{Tr} \mathbf{H}_{1}^{2}\right\}$, and assume $\phi$ to be positive without any loss of generality. Under these assumptions, the connected part of the parametric level densities correlator defined as

$$
\begin{aligned}
R_{N}^{(c)}\left(E, E^{\prime} ; \phi\right) & =\left\langle\operatorname{Tr} \delta\left(E-\mathbf{H}_{0}\right) \operatorname{Tr} \delta\left(E^{\prime}-\mathbf{H}_{\phi}\right)\right\rangle \\
& -\left\langle\operatorname{Tr} \delta\left(E-\mathbf{H}_{0}\right)\right\rangle\left\langle\operatorname{Tr} \delta\left(E^{\prime}-\mathbf{H}_{\phi}\right)\right\rangle,
\end{aligned}
$$

where the angular brackets $\langle\ldots\rangle$ stand for averaging over the ensembles of random matrices $\mathbf{H}_{0}$ and $\mathbf{H}_{1}$, is given by

$$
\begin{aligned}
R_{N}^{(c)} & \left(E, E^{\prime} ; \phi\right) \equiv-\frac{1}{\pi} \int_{-\infty}^{+\infty} d \sigma_{1} \int_{-\infty}^{+\infty} d \sigma_{2} \mathrm{e}^{-\left(\sigma_{1}^{2}+\sigma_{2}^{2}\right)} \\
& \times \mathrm{e}^{\frac{1}{2}\left[v\left(E^{\prime}+i \phi \sigma_{1}\right)-v\left(E^{\prime}+\phi \sigma_{2}\right)\right]} K_{N}\left(E, E^{\prime}+i \phi \sigma_{1}\right) \\
& \times\left[K_{N}\left(E^{\prime}+\phi \sigma_{2}, E\right)-\delta\left(E^{\prime}+\phi \sigma_{2}-E\right)\right]
\end{aligned}
$$

Equation (4) is exact for arbitrary finite $N$, and the sum rule for $R_{N}^{(c)}$ is fulfilled by construction. Note, that for finite $N$, the equation contains both the two-point kernel $K_{N}\left(E, E^{\prime}\right)$ of an unperturbed matrix ensemble Eq.
(11) and the confinement potential $v(E)$. It is worth mentioning that only for the GUE measure, $v\left[\mathbf{H}_{0}\right] \propto \mathbf{H}_{0}^{2}$, this finite $-N$ solution reduces to the one known from the Dyson Brownian motion model [18] in the limit $\phi \ll 1$.

Thermodynamic limit $N \rightarrow \infty$ of Eq. (4) is substantially different in the case of the soft and the strong level confinement. (i) If the confinement potential is soft, the two-point kernel $K_{N}$ has a well defined large- $N$ limit given by a corresponding Poisson kernel $K_{P}\left(E, E^{\prime} ; r\right)=$ $\sum_{n=0}^{\infty} r^{n} \psi_{n}(E) \psi_{n}\left(E^{\prime}\right)$ taken at $r=1$ [19]. As a consequence, Eq. (4) holds in the large $-N$ limit as it stands, with $K_{N}$ replaced by $K_{P}(r=1)$. After implementation of $N$-independent unfolding, $E \mapsto s, \phi \mapsto X$, it provides an exact answer. Hence, generically there are no reasons to expect a universality of parametric level statistics in ensembles with soft level confinement. (ii) For strong level confinement, Eq. (4) can take a universal form in the thermodynamic limit after the implementation of the unfolding procedure $d s / d E=\bar{\nu}_{N}, X=\phi \bar{\nu}_{N}$ (with $\bar{\nu}_{N}$ being the local level density [20]),

$$
\begin{aligned}
R^{(c)} & \left(s, s^{\prime} ; X\right)=-\frac{1}{\pi} \int_{-\infty}^{+\infty} d \sigma_{1} \int_{-\infty}^{+\infty} d \sigma_{2} \mathrm{e}^{-\left(\sigma_{1}^{2}+\sigma_{2}^{2}\right)} \\
& \times F_{X}\left(s^{\prime}, \sigma_{1}, \sigma_{2}\right) K\left(s, s^{\prime}+i X \sigma_{1}\right) \\
& \times\left[K\left(s^{\prime}+X \sigma_{2}, s\right)-\delta\left(s^{\prime}+X \sigma_{2}-s\right)\right]
\end{aligned}
$$

where

$$
F_{X}\left(s, \sigma_{1}, \sigma_{2}\right)=\lim _{N \rightarrow \infty} \frac{\exp \left\{\frac{1}{2}\left[v\left(E_{N, s}+i \phi_{N, X} \sigma_{1}\right)\right]\right\}}{\exp \left\{\frac{1}{2}\left[v\left(E_{N, s}+\phi_{N, X} \sigma_{2}\right)\right]\right\}} .
$$

Equations (5) and (6) establish a link between the parametric level statistics in unitary invariant non-Gaussian ensemble of random matrices and the energy level statistics in the same, unperturbed random matrix ensemble, through the unfolded two-point kernel

$$
\begin{aligned}
K\left(s, s^{\prime}\right) & \equiv \lim _{N \rightarrow \infty} K_{N}\left(E_{N, s}, E_{N, s^{\prime}}\right) \\
& \times \nu_{N}^{-1 / 2}\left(E_{N, s}\right) \nu_{N}^{-1 / 2}\left(E_{N, s^{\prime}}\right) .
\end{aligned}
$$

Connection relations Eqs. (4) - (7) provide a constructive rule for computing parametric level statistics in nonGaussian matrix models and constitute the main results of the paper.

In particular, when both $F_{X}$ and $K\left(s, s^{\prime}\right)$ are universal functions for a class of matrix models, the universality of eigenlevel correlations will automatically imply the universality of parametric level correlations. To demonstrate this point, consider the matrix model Eq. (1) with strong confinement potential exhibiting a logarithmic singularity, $v(E) \rightarrow v(E)-2 \alpha \log |E|(\alpha>-1 / 2)$. For $\beta=2$ and in the absence of the perturbation, this model has been proven to possess three different types of locally universal eigenlevel correlations in the bulk (BSL) [21], the origin (OSL) 22,9] and the soft-edge (SSL) scaling limits [9]. In all these scaling limits, the two-point kernel obeys the universal laws which we convert to 


$$
K\left(s, s^{\prime}\right)=\frac{\Psi_{1}(s) \Psi_{2}\left(s^{\prime}\right)-\Psi_{2}(s) \Psi_{1}\left(s^{\prime}\right)}{s-s^{\prime}} .
$$

Here $s$ and $s^{\prime}$ are appropriately unfolded spectral variables,

$$
\Psi_{1}(s)=\left\{\begin{array}{cc}
\pi^{-1} \sin (\pi s), & \text { BSL, } \\
-(s / 2 \pi)^{1 / 2} J_{\alpha-1 / 2}(\pi s), & \text { OSL, } \\
\operatorname{Ai~}(s), & \text { SSL }
\end{array}\right.
$$

$\Psi_{2}(s)=\Psi_{1}^{\prime}(s)$ for BSL and SSL, while for OSL $\Psi_{2}(s)=$ $\Psi_{1}^{\prime}(s)-\alpha s^{-1} \Psi_{1}(s)$. These three universal kernels are referred to as the sine, the Bessel, and the Airy kernels, respectively. Universal parametric level statistics in this ensemble is a consequence of the connection relations Eqs. (5) and (6), which enable us to write down closed analytical expressions for the parametric level density correlator $R^{(c)}$ just by noting that $F_{X}$ is a universal function: $F_{X}\left(s, \sigma_{1}, \sigma_{2}\right)=\left(s+X \sigma_{2}\right)^{\alpha}\left(s+i X \sigma_{1}\right)^{-\alpha}$ in the origin scaling limit, and $F_{X}=1$ otherwise.

Let us now outline the derivation of the announced relations. The two-point parametric correlator $R_{N}\left(E, E^{\prime} ; \phi\right)$ of level densities can be represented as an average over the joint distribution function

$$
\mathcal{P}_{2}\left[\mathbf{H}_{0}, \mathbf{H}_{\phi}\right] \propto \exp \left\{-\operatorname{Tr}\left(v\left[\mathbf{H}_{0}\right]+\phi^{-2}\left(\mathbf{H}_{0}-\mathbf{H}_{\phi}\right)^{2}\right)\right\},
$$

of two Hamiltonians, unperturbed $\mathbf{H}_{0}$ and perturbed $\mathbf{H}_{\phi}$,

$$
R_{N}\left(E, E^{\prime} ; \phi\right)=\left\langle\operatorname{Tr} \delta\left(E-\mathbf{H}_{0}\right) \operatorname{Tr} \delta\left(E^{\prime}-\mathbf{H}_{\phi}\right)\right\rangle_{\mathcal{P}_{2}} .
$$

In Eq. (11) the averaging over the distribution $\mathcal{P}_{2}$ can explicitly be performed by using the Eynard-Mehta theorem [23]. The theorem states that $R_{N}\left(E, E^{\prime} ; \phi\right)$ equals the determinant of the $2 \times 2$ matrix kernel,

$$
R_{N}\left(E, E^{\prime} ; \phi\right)=\operatorname{Det}\left[k_{\alpha \beta}^{(N)}\left(E_{\alpha}, E_{\beta}\right)\right]_{\alpha, \beta=1,2},
$$

where $E_{\alpha}=E+\left(E^{\prime}-E\right)\left[1+(-1)^{\alpha}\right] / 2$. An explicit representation of the matrix kernel reads,

$$
\begin{aligned}
k_{\alpha \beta}^{(N)}\left(\xi, \xi^{\prime}\right)= & \phi^{-1} \sum_{n=0}^{N-1} h_{n}^{-1} P_{\alpha, n}\left(\frac{\xi}{\phi}\right) Q_{\beta, n}\left(\frac{\xi^{\prime}}{\phi}\right) \\
& -\phi^{-1} \delta_{\alpha, \beta-1} W\left(\frac{\xi}{\phi}, \frac{\xi^{\prime}}{\phi}\right) .
\end{aligned}
$$

Here $P_{\alpha, n}$ and $Q_{\alpha, n}$ are four complete sets of orthogonal functions; two of them, $P_{1, n}=P_{n}$ and $Q_{2, n}=Q_{n}$, are basic polynomials orthogonal with respect to the nonlocal weight $W(\xi, \eta)=\exp \left\{-v(\phi \xi)-(\xi-\eta)^{2}\right\}$,

$$
\int d \xi \int d \eta P_{n}(\xi) W(\xi, \eta) Q_{m}(\eta)=h_{n} \delta_{n m}
$$

with $h_{n}$ being a normalization constant. (Hereafter, integration over single variable runs over entire real axis).
Two remaining orthogonal functions are connected to the basic polynomials as follows:

$$
\begin{aligned}
P_{2, n}(\xi) & =\int d \eta P_{n}(\eta) W(\eta, \xi), \\
Q_{1, n}(\xi) & =\int d \eta W(\xi, \eta) Q_{n}(\eta) .
\end{aligned}
$$

Remarkably, all of the four sets $P_{\alpha, n}$ and $Q_{\alpha, n}$ can solely be expressed through the orthogonal polynomials $p_{n}(E)$, Eq. (2), which determine the eigenlevel statistics in an unperturbed random matrix ensemble Eq. (11). To prove this, we use the representation [24 of polynomials $P_{n}$ and $Q_{n}$ orthogonal with respect to a nonlocal measure $W$ as a determinant averaged over the distribution $\mathcal{P}_{2}\left[\phi \mathbf{M}_{1}, \phi \mathbf{M}_{2}\right]$, where $\mathbf{M}_{1}$ and $\mathbf{M}_{2}$ are $n \times n$ Hermitian matrices:

$$
\begin{aligned}
P_{n}(\xi) & =\left\langle\operatorname{Det}_{n \times n}\left(\xi-\mathbf{M}_{1}\right)\right\rangle_{\mathcal{P}_{2}\left[\phi \mathbf{M}_{1}, \phi \mathbf{M}_{2}\right]}, \\
Q_{n}(\xi) & =\left\langle\operatorname{Det}_{n \times n}\left(\xi-\mathbf{M}_{2}\right)\right\rangle_{\mathcal{P}_{2}\left[\phi \mathbf{M}_{1}, \phi \mathbf{M}_{2}\right]} .
\end{aligned}
$$

Integration over matrix $\mathbf{M}_{2}$ in Eq. (16a) is straightforward; it yields the expression

$$
P_{n}(\xi)=\left\langle\operatorname{Det}_{n \times n}\left(\xi-\mathbf{M}_{1}\right)\right\rangle_{\mathcal{P}_{1}\left[\phi \mathbf{M}_{1}\right]}=\phi^{1 / 2} p_{n}(\phi \xi),
$$

which we have recognized as a matrix-integral representation 25] of the ordinary orthogonal polynomials appearing in Eq. (2). To integrate out the matrix $\mathbf{M}_{2}$ in Eq. (16b) we rewrite the determinant there in the form of the integral $\operatorname{Det}_{n \times n}\left(\xi-\mathbf{M}_{2}\right)=$ $\int D[\chi] \exp \left\{-\chi^{\dagger}\left(\xi-\mathbf{M}_{2}\right) \chi\right\}$ over $n$-component Grassmann vector $\chi=\left(\chi_{1}, \ldots, \chi_{n}\right)$. Further integration over $\mathbf{M}_{2}$ leads to

$$
\begin{aligned}
Q_{n}(\xi)= & \int D[\chi] \exp \left\{-\frac{1}{4}\left(\chi^{\dagger} \chi\right)^{2}\right\} \\
& \times\left\langle\exp \left\{-\chi^{\dagger}\left(\xi-\mathbf{M}_{1}\right) \chi\right\}\right\rangle_{\mathcal{P}_{1}\left[\phi \mathbf{M}_{1}\right]} .
\end{aligned}
$$

Decoupling the 'interaction' term $\exp \left\{-\left(\chi^{\dagger} \chi\right)^{2} / 4\right\}$ in Eq. (18) followed by integration over the Grassmann vector $\chi$ results in

$$
Q_{n}(\xi)=\pi^{-1 / 2} \int d \sigma_{1} \mathrm{e}^{-\sigma_{1}^{2}} P_{n}\left(\xi+i \sigma_{1}\right)
$$

Combining Eqs. (15), (17) and (19), we obtain explicit expressions for $P_{\alpha, n}$ and $Q_{\alpha, n}$ entering Eq. (13) for the matrix kernel $k_{\alpha \beta}^{(N)}$,

$$
\begin{aligned}
P_{1, n}(\xi) & =\phi^{1 / 2} p_{n}(\phi \xi) \\
P_{2, n}(\xi) & =\phi^{1 / 2} \int d \eta W(\xi, \eta) p_{n}(\phi \eta), \\
Q_{1, n}(\xi) & =(\pi \phi)^{1 / 2} \mathrm{e}^{-v(\phi \xi)} p_{n}(\phi \xi) \\
Q_{2, n}(\xi) & =\left(\pi^{-1} \phi\right)^{1 / 2} \int d \sigma_{1} \mathrm{e}^{-\sigma_{1}^{2}} p_{n}\left(\phi\left(\xi+i \sigma_{1}\right)\right) .
\end{aligned}
$$


One can verify that the orthogonality relation Eq. (14) is fulfilled, and the normalization constants in Eqs. (2) and (14) are related as $h_{n}=\pi^{-1 / 2} \mathcal{N}_{n}$. Substituting Eqs. (20) into Eqs. (13) and (12), and subtracting disconnected part given by the product $k_{11}^{(N)} k_{22}^{(N)}$ is the final stage of the calculations which leads to our solution Eq. (仿). Equations (5) and (6) follow upon appropriate unfolding. Note that these results can be extended to the $n$-point parametric correlators along the lines of Ref. [26.

In conclusion, we presented an exact solution, Eq. (4), to the problem of parametric level statistics in unitary invariant, non-Gaussian random matrix ensembles characterized by either soft or strong level confinement. The solution is exact in precisely the same sense as are the formulas by Gaudin and Mehta [2] for conventional spectral statistics. Generically, the parametric 'density-density' correlator was shown to depend on both the two-point scalar kernel and the level confinement, through a double integral transformation which, in turn, provides a constructive tool for description of parametric level correlations in non-Gaussian random matrix theory. In random matrix ensembles with strong level confinement, the solution presented takes a particular simple form in the thermodynamic limit leading to emergence of a new connection relation, Eq. (5), between the parametric spectral statistics and the scalar two-point kernel of an unperturbed ensemble. In the case of soft level confinement, the formalism developed is potentially applicable to a study of parametric level statistics in unitary invariant random matrix model with log-squared confinement potential [6], $v(E) \propto \log ^{2}|E|$ at $|E| \gg 1$. The latter problem is of conceptual interest in view of the recent work [7] where existence of a new class of random matrix ensembles with finite eigenlevel compressibility and multifractal eigenvectors was suggested. There, identical two-level correlation functions in proper regimes in three different ensembles - (a) generalized matrix model of Ref. [27], (b) power-law banded matrices of Ref. [28], and (c) rotationally invariant random matrices with logsquared confinement potential already mentioned above - were one of the key points to identifying the new universality class relevant to the one expected at the edge of the metal-insulator transition. In this context, it would be desirable to learn whether these three matrix models also enjoy the same parametric level statistics. At least for two of the ensembles [(b) and (c)] such a verification seems now to be possible.

I thank V. E. Kravtsov, K. A. Muttalib and I. Yurkevich for discussions at different stages of this study.

[1] T. Guhr, A. Müller-Groeling, and H. A. Weidenmüller, Phys. Rep. 299, 189 (1998).

[2] M. L. Mehta, Random Matrices (Academic Press, Boston, 1991).

[3] J. J. M. Verbaarschot, Universal Fluctuations in Dirac Spectra, in: New Developments in Quantum Field The- ory, edited by P. H. Damgaard and J. Jurkiewicz, NATO ASI, Ser. B, Vol. 366 (Plenum Press, New York, 1998).

[4] A. Altland and M. R. Zirnbauer, Phys. Rev. B 55, 1142 (1997).

[5] T. Nagao and K. Slevin, Phys. Rev. Lett. 70, 635 (1993).

[6] K. A. Muttalib, Y. Chen, M. E. H. Ismail, and V. N. Nicopoulos, Phys. Rev. Lett. 71, 471 (1993); C. M. Canali and V. E. Kravtsov, Phys. Rev. E 51, R5185 (1995); E. Bogomolny, O. Bohigas, and M. P. Pato, Phys. Rev. E 55, 6707 (1997); S. M. Nishigaki, Phys. Rev. E 58, R6915 (1998).

[7] V. E. Kravtsov and K. A. Muttalib, Phys. Rev. Lett. 79, 1913 (1997).

[8] The confinement potential $v(E)$ in Eq. (1) is said to be 'strong', if it increases at least as $|E|$ at infinity; otherwise it is referred to as 'soft' level confinement.

[9] E. Kanzieper and V. Freilikher, Phys. Rev. Lett. 78, 3806 (1997); Philos. Mag. B 77, 1161 (1998); Phys. Rev. E 57 6604 (1998).

[10] E. Kanzieper and V. Freilikher, Spectra of large random matrices: A method of study, in: Diffuse Waves in Complex Media, edited by J.-P. Fouque, NATO ASI, Ser. C, Vol. 531 (Kluwer, Dordrecht, 1999).

[11] G. Akemann, P. H. Damgaard, U. Magnea, and S. Nishigaki, Nucl. Phys. B 519, 682 (1998).

[12] M. K. Şener and J. J. M. Verbaarschot, Phys. Rev. Lett. 81, 248 (1998).

[13] H. Widom, eprint solv-int/9804005 (1998).

[14] E. Brézin and H. Neuberger, Nucl. Phys. B 350, 513 (1991).

[15] C. A. Tracy and H. Widom, J. Stat. Phys. 92, 809 (1998).

[16] E. Brézin and A. Zee, Phys. Rev. E 49, 2588 (1994). These authors address the universality of so-called smoothed parametric correlators for $\beta=2$.

[17] G. Hackenbroich and H. A. Weidenmüller, Phys. Rev. Lett. 74, 4118 (1995).

[18] F. Dyson, J. Math. Phys. 3, 1191 (1962); C. W. J. Beenakker and B. Rejaei, Physica A 203, 61 (1994).

[19] Due to a lack of completeness property of $\left\{\psi_{n}\right\}$ in this case, the Poisson kernel $K_{P}\left(E, E^{\prime} ; r=1\right)$ is no longer reduced to $\delta\left(E-E^{\prime}\right)$. See, for instance: M. E. H. Ismail and D. R. Masson, Trans. Am. Math. Soc. 346, 63 (1994) and references therein.

[20] In the origin and the bulk scaling limits, $\bar{\nu}_{N} \equiv \nu_{N}$. In the soft-edge scaling limit, $\bar{\nu}_{N}$ is defined as $\bar{\nu}_{N}=$ $\left(D_{N} / 2\right)^{1 / 2} \lim _{t \rightarrow+0}\left[t^{-1 / 2} \nu_{N}\left(D_{N}-t\right)\right], D_{N}$ being the end point of the spectrum. For further details, see Ref. [10].

[21] E. Brézin and A. Zee, Nucl. Phys. B 402, 613 (1993).

[22] G. Akemann, P. H. Damgaard, U. Magnea, and S. Nishigaki, Nucl. Phys. B 487, 721 (1997).

[23] B. Eynard and M. L. Mehta, J. Phys. A 31, 4449 (1998).

[24] B. Eynard, J. Phys. A 31, 8081 (1998).

[25] G. Szegö, Orthogonal Polynomials (American Mathematical Society, Providence, 1967).

[26] E. Kanzieper and V. Freilikher, Phys. Rev. E 59, 3720 (1999).

[27] M. Moshe, H. Neuberger, and B. Shapiro, Phys. Rev. Lett. 73, 1497 (1994).

[28] A. D. Mirlin, Y. V. Fyodorov, F.-M. Dittes, J. Quezada, and T. H. Seligman, Phys. Rev. E 54, 3221 (1996). 